\title{
A new deformation theory with strain gradient effects
}

\author{
S.H. Chen*, T.C. Wang \\ LNM, Institute of Mechanics, Chinese Academy of Sciences, Beijing, 100080, China
}

Received in final revised form 3 February 2001

\begin{abstract}
A new phenomenological deformation theory with strain gradient effects is proposed. This theory, which belongs to nonlinear elasticity, fits within the framework of general couple stress theory and involves a single material length scale $l$. In the present theory three rotational degrees of freedom $\omega_{i}$ are introduced in addition to the conventional three translational degrees of freedom $u_{i}$. $\omega_{i}$ has no direct dependence upon $u_{i}$ and is called the micro-rotation, i.e. the material rotation $\theta_{i}$ plus the particle relative rotation. The strain energy density is assumed to only be a function of the strain tensor and the overall curvature tensor, which results in symmetric Cauchy stresses. Minimum potential principle is developed for the strain gradient deformation theory version. In the limit of vanishing $l$, it reduces to the conventional counterparts: $J_{2}$ deformation theory. Equilibrium equations, constitutive relations and boundary conditions are given in details. Comparisons between the present theory and the theory proposed by Shizawa and Zbib (Shizawa, K., Zbib, H.M., 1999. A thermodynamical theory gradient elastoplasticity with dislocation density Censor: fundamentals. Int. J. Plast. 15, 899) are given. With the same hardening law as Fleck et al. (Fleck, N.A., Muller, G.H., Ashby, M.F., Hutchinson, J.W., 1994 Strain gradient plasticity: theory and experiment. Acta Metall. Mater 42, 475), the new strain gradient deformation theory is used to investigate two typical examples, i.e. thin metallic wire torsion and ultra-thin metallic beam bend. The results are compared with those given by Fleck et al, 1994 and Stolken and Evans (Stolken, J.S., Evans, A.G., 1998. A microbend test method for measuring the plasticity length scale. Acta Mater. 46, 5109). In addition, it is explained for a unit cell that the overall curvature tensor produced by the overall rotation vector is the work conjugate of the overall couple stress tensor. (C) 2002 Elsevier Science Ltd. All rights reserved.
\end{abstract}

Keywords: Microstructure; Strain gradient effects; Couple stress theory; Constitutive behavior; Nonlinear elasticity

* Corresponding author.

E-mail address: chenshaohua72@hotmail.com (S.H. Chen). 


\section{Introduction}

Many experiments have shown that materials display strong size effects when the characteristic length scale associated with non-uniform plastic deformation is on the order of microns. For an aluminum-silicon matrix reinforced by silicon carbide particles, Lloyd (1994) observed a substantial strength increase when the particle diameter was reduced from 16 to $7.5 \mu \mathrm{m}$ with the particle volume fraction fixed at $15 \%$. In the experiments of measuring micro-indentation hardness of metallic materials the square of the hardness increases linearly as the depth of indentation decreases (Nix, 1989; Ma and Clarke, 1995; Poole et al., 1996; McElhaney et al., 1998). The overall properties of a polycrystal are functions of $l / a$, which reflects the grain size effect, where $l$ is the intrinsic length scale introduced into crystal's constitutive law by the gradient effects and $a$ is the grain size (Smyshlyaev and Fleck, 1996). The most compelling experimental evidences that strong size effects exist have been provided by Fleck et al. (1994) and Stolken and Evans (1998). The former is to measure the scaled shear strength while twisting thin wires with different diameters and the latter is to measure the bend moments while bending ultra-thin beams with different thickness.

In the fracture problem, Elssner et al. (1994) did a series of experiments and measured both the macroscopic fracture toughness and atomic work of separation of an interface between a single crystal of niobium and a sapphire single crystal. They found that the interface between the two materials remained atomistically sharp, i.e. the crack tip was not blunted even though niobium is ductile and has a large number of dislocations. The stress level needed to produce atomic decohesion of a lattice is on the order of 10 times the tensile yield stress. Hutchinson (1997) pointed out that the maximum stress level that can be achieved near a crack tip is not larger than 4-5 times the tensile yield stress of metals, according to models based on conventional plasticity theories.

Recent years several theories (Dillon et al., 1970; Fleck and Hutchinson, 1993, 1997; Fleck et al., 1994; Nilsson, 1998; Gao et al., 1999; Shu and Fleck, 1999) about strain gradient effects were proposed. The strain gradient crystal formulation proposed by Shu and Fleck (1999) fits within the Fleck and Hutchinson (1997) plasticity theory framework and has been used to analyze the scale-dependence of strength and deformation in a porous single crystal (Shu, 1998) and microscopic strain field in a metal matrix composite (Shu and Barlow, 2000). Fleck and Hutchinson (1993) and Fleck et al. (1994) developed a deformation theory version of plasticity, which models the hardening due to both statistically stored and geometrically necessary dislocations. Their theory fits neatly within the general framework of reduced couple stress theory and reduces to conventional $J_{2}$ deformation theory in the absence of strain gradient effects. In comparison, no work conjugate of strain gradient has been defined in the alternative gradient theories (Aifantis, 1984,1987; Muhlhaus and Aifantis, 1991) which represent the strain gradient effects as terms relative with Laplacian of effective strain. Retaining the essential structure of conventional plasticity and obeying thermodynamic restrictions, Acharya and Bassani (1995) conclude that the only possible formulation is a flow theory with strain gradient effects 
represented as an internal variable, which acts to increase the current tangent-hardening modulus. However, there has not been a systematic way of constructing the tangent modulus so as to validate this framework.

In 1999, Shizawa and Zbib developed a thermodynamical theory of gradient elastoplasticity by introducing the concept of dislocation density tensor. In their theory, the microstress is conjugate to the dislocation density tensor; moreover the stress tensor is symmetric, which is same as that in this paper. The comparison between the theory proposed by Shizawa and Zbib (1999) and the present theory will be shown in the context.

Chen and Wang (2000) established a concrete hardening law based on the incremental version of conventional $J_{2}$ deformation theory, in which the effective strain gradient is only a parameter to influence the tangent modulus.

In the present paper, the deformation theory version of a new strain gradient theory is proposed based on the general couple stress theory. Minimum potential principle is established for solving boundary value problems. Two typical phenomena, i.e. thin metallic wire torsion and ultra-thin metallic beam bend, are investigated using the new strain gradient theory. The results exhibit that if the boundary conditions are properly taken in the new theory, the solutions to the two examples will be the same as that in Fleck et al. (1994), Stolken and Evans (1998). Furthermore, using the new strain gradient theory, the hydrostatic part of the couple stress tensor can be obtained so that the boundary conditions of moments on the lateral faces can be exactly met and it enables the use of $C_{0}$ continuous elements in a finite element formation.

Review of the general couple stress theory is given in Section 2. The new strain gradient deformation theory extended from the general couple stress theory is given in Section 3. Comparison between the thermodynamical theory proposed by Shizawa and Zbib (1999) and the new theory in the present paper will be given in Section 4. In Section 5, two typical problems are analyzed using the new strain gradient deformation theory in the present paper and the results are compared with that obtained using the theory of Fleck et al. (Fleck and Hutchinson, 1993; Fleck et al., 1994). Strain gradient flow theory version is briefly introduced in Section 6.

\section{Review of the general couple stress theory}

In the general couple stress theory, the work-conjugated rotation, $\boldsymbol{\omega}$, is treated as an independent kinematic quantity with no direct dependence upon $\mathbf{u}$ and distinct from the associated rotation $\boldsymbol{\theta} \equiv(1 / 2)$ curlu. $\boldsymbol{\sigma}$ denotes the symmetric part of Cauchy stress and $\tau$ denotes the anti-symmetric part of Cauchy stress, $\mathbf{m}$ denotes the overall couple stress tensor.

Neglecting the body forces and body couples, the principle of virtual work for the general couple stress theory is

$$
\int_{V}\left[\left(\sigma_{i j}+\tau_{i j}\right) \delta \gamma_{i j}+m_{i j} \delta \chi_{i j}\right] \mathrm{d} V=\int_{S}\left[T_{i} \delta u_{i}+q_{i} \delta \omega_{i}\right] \mathrm{d} S
$$


where $\chi_{i j}=\omega_{i, j}$ is the work-conjugated curvature tensor and $\gamma_{i j}=u_{i, j}+e_{i j k} \omega_{k}$ is called the relative displacement gradient tensor. $V$ is the volume of the body and $S$ is the surface of it.

The above virtual work statement can be rearranged to the form

$$
\int_{V}\left(\sigma_{i j} \delta \varepsilon_{i j}+\tau_{i j} \delta \alpha_{i j}+m_{i j} \delta \chi_{i j}\right] \mathrm{d} V=\int_{S}\left[T_{i} \delta u_{i}+q_{i} \delta \omega_{i}\right] \mathrm{d} S
$$

where the symmetric tensor $\varepsilon_{i j}$ is the usual strain tensor,

$$
\varepsilon_{i j}=\left(u_{i, j}+u_{j, i}\right) / 2
$$

and the anti-symmetric tensor $\alpha_{i j}$ is the relative rotation tensor

$$
\alpha_{i j}=e_{i j k} \omega_{k}-\left(u_{j, i}-u_{i, j}\right) / 2=e_{i j k}\left(\omega_{k}-\theta_{k}\right)
$$

Via the divergence theorem, the left in Eq. (2) can be written

$$
\begin{aligned}
\int_{V}\left(\sigma_{i j} \delta \varepsilon_{i j}+\tau_{i j} \delta \alpha_{i j}+m_{i j} \delta \chi_{i j}\right) \mathrm{d} V= & \int_{S}\left(\sigma_{i j}+\tau_{i j}\right) n_{j} \delta u_{i} \mathrm{~d} S-\int_{V}\left(\sigma_{i j}+\tau_{i j}\right)_{, j} \delta u_{i} \mathrm{~d} V \\
& +\int_{S} m_{i j} n_{j} \delta \omega_{i} \mathrm{~d} S+\int_{V} \tau_{j k} e_{i j k} \delta \omega_{i} \mathrm{~d} V \\
& -\int_{V} m_{i j, j} \delta \omega_{i} \mathrm{~d} V
\end{aligned}
$$

then, the equilibrium relation of force in $V$ is

$$
t_{i j, j}=\sigma_{i j, j}+\tau_{i j, j}=0
$$

and moment equilibrium is

$$
\tau_{j k}=\frac{1}{2} e_{i j k} m_{i p, p}
$$

Here, a comma indicates a partial derivative with respect to a Cartesian coordinate and a repeated suffix denotes summation over 1-3. A subscript index can take the value of 1,2 or 3 and $e_{i j k}$ denotes the usual permutation symbol.

Traction equilibrium on the surface $S$ of the body is

$$
T_{i}=\left(\sigma_{i j}+\tau_{i j}\right) n_{j}, \quad q_{i}=m_{i j} n_{j}
$$

The strain energy density function $w$ in the general couple stress theory depends upon the strain tensor $\varepsilon$, the curvature tensor $\chi$ and the relative rotation $\alpha$ as following

$$
w(\varepsilon, \boldsymbol{\chi}, \boldsymbol{\alpha})=\int_{0}^{\varepsilon_{i j}} \sigma_{i j} \mathrm{~d} \varepsilon_{i j}+\int_{0}^{\chi_{i j}} m_{i j} \mathrm{~d} \chi_{i j}+\int_{0}^{\alpha_{i j}} \tau_{i j} \mathrm{~d} \alpha_{i j}
$$


then, the constitutive relations are

$$
\sigma_{i j}=\frac{\partial w}{\partial \varepsilon_{i j}}, m_{i j}=\frac{\partial w}{\partial \chi_{i j}}, \tau_{i j}=\frac{\partial w}{\partial \alpha_{i j}}
$$

\section{The new strain gradient deformation theory}

There are a lot of works about couple stress theory, such as the works of Toupin (1962), Mindlin (1963, 1964), Schaefer (1967) and Eringen (1968). Specially, Green et al. (1968) proposed a dipolar theory of plasticity in the presence of simple force and stress dipoles. Also Naghdi and Srinivasa (1993a,b, 1994) developed a Cosserat theory (Cosserat and Cosserat, 1909) with three directors and solved problems involving the evolution of dislocations. All these theories are based on a reduced couple stress theory model, in which the rotation vector is related with the displacement vector, $\theta \equiv(1 / 2)$ curlu, and then the relative rotation tensor $\alpha_{i j}$ vanishes.

The present strain gradient deformation theory is proposed for the materials containing numerous micro dispersion particles or micro precipitation particles and based on the framework of general couple stress theory. The size of these particles is very small and about $10 \mathrm{~nm}$. An idealized model for such kind of material is that the particles are compact and the materials are full of these particles. In a deformation theory there is no formal distinction between the elastic and plastic components of displacement and of strain, then we write $\varepsilon_{e}=\sqrt{\frac{2}{3} \varepsilon_{i j} \varepsilon_{i j}}$ and $\chi_{e}=\sqrt{\frac{2}{3} \chi_{i j} \chi_{i j}}$. Particle's

rotation is denoted by the micro-rotation vector $\boldsymbol{\omega}$, which is the sum of material rotation and the particle relative rotation. $\boldsymbol{\omega}$ is an independent quantity with no direct dependence upon $\mathbf{u}$ and $\boldsymbol{\omega} \neq \boldsymbol{\theta}$, so that the particle relative rotation is not equal zero.

We should emphasize that the curvature tensor $\chi_{i j}$ not $\theta_{i, j}$ is the work conjugate of the couple stress $m_{i j}$, the detail discussion is provided in appendix A.

We postulate that the strain energy density $w$ depends only upon the strain tensor $\varepsilon$ and the curvature tensor $\chi$, i.e. the relative rotation tensor $\alpha$ has no contribution to the strain energy density $w$. From Eq. (10), it follows

$$
\tau_{i j}=\frac{\partial w}{\partial \alpha_{i j}}=0
$$

Consider a body of volume $V$ and surface $S$ comprised of non-linear elastic solid: the solid satisfied the constitutive law Eq. (10). Stress traction $T_{i}^{0}$ acts on a portion $S_{T}$ of the surface of the body, on the remaining portions $S_{u}$ the displacement is prescribed as $u_{i}^{0}$ and couple stress traction $q_{i}^{0}$ acts on a portion $S_{q}$, on the remaining portions $S_{\omega}$ of the surface the rotation is prescribed as $\omega_{i}^{0}$. 
The equilibrium equations for stress and couple stress in the body become

$$
\sigma_{i j, j}=0 \quad m_{i j, j}=0
$$

The traction boundary conditions for force and moment are

$$
\begin{array}{ll}
\sigma_{i j} n_{j}=T_{i}^{0} & \text { on } S_{T} \\
m_{i j} n_{j}=q_{i}^{0} & \text { on } S_{q}
\end{array}
$$

The additional boundary conditions are

$$
\begin{array}{llr}
u_{i}=u_{i}^{0} & \text { on } & S_{u} \\
\omega_{i}=\omega_{i}^{0} & \text { on } & S_{\omega}
\end{array}
$$

The deviatoric part $s_{i j}$ of Cauchy stress and deviatoric part $m_{i j}^{\prime}$ of couple stress are defined as the work conjugates of $\varepsilon_{i j}^{\prime}, \chi_{i j}^{\prime}$ respectively; $\sigma_{m}$ and $m_{m}$ are defined as the work conjugates of $\varepsilon_{m}$ and $\chi_{m}$ respectively, giving

$$
\delta w=s_{i j} \delta \varepsilon_{i j}^{\prime}+m_{i j}^{\prime} \delta \chi_{i j}^{\prime}+\sigma_{m} \delta \varepsilon_{m}+m_{m} \delta \chi_{m}
$$

where $s_{i j} \equiv \sigma_{i j}-(1 / 3) \delta_{i j} \sigma_{k k}$ and $m_{i j}^{\prime} \equiv m_{i j}-(1 / 3) \delta_{i j} m_{k k}$.

Eq. (17) enables one to determine $s_{i j}, m_{i j}^{\prime}, \sigma_{m}$ and $m_{m}$ in terms of the strain and curvature states of the solid as

$$
s_{i j}=\frac{\partial w}{\partial \varepsilon_{i j}^{\prime}}, \quad m_{i j}^{\prime}=\frac{\partial w}{\partial \chi_{i j}^{\prime}}, \quad \sigma_{m}=\frac{\partial w}{\partial \varepsilon_{m}}, \quad m_{m}=\frac{\partial w}{\partial \chi_{m}}
$$

According to the work by Fleck and Hutchinson (1993) and Fleck et al. (1994), it is mathematically convenient to assume that the strain energy density $w$ depends only upon the single scalar strain measure $E_{e}$, where

$$
E_{e}^{2}=\varepsilon_{e}^{2}+l^{2} \chi_{e}^{2}
$$

where the length scale $l$ is a material length scale, and is required on dimensional grounds.

An effective stress measure $\Sigma_{e}$ is defined as the work conjugate of $E_{e}$,

$$
\Sigma_{e}=\frac{\mathrm{d} w\left(E_{e}\right)}{\mathrm{d} E_{e}}
$$

then

$$
\begin{aligned}
& s_{i j}=\frac{\partial w}{\partial \varepsilon_{i j}^{\prime}}=\frac{2 \Sigma_{e}}{3 E_{e}} \varepsilon_{i j}^{\prime}, \quad m_{i j}^{\prime}=\frac{2}{3} l^{2} \frac{\Sigma_{e}}{E_{e}} \chi_{i j}^{\prime}, \quad \sigma_{m}=\frac{1}{3} \sigma_{k k}, \quad m_{m}=\frac{1}{3} m_{k k} \\
& \Sigma_{e}=\left(\sigma_{e}^{2}+l^{-2} m_{e}^{2}\right)^{1 / 2}
\end{aligned}
$$


where

$$
\begin{cases}\sigma_{e}^{2}=\frac{3}{2} s_{i j} s_{i j}, & m_{e}^{2}=\frac{3}{2} m_{i j}^{\prime} m_{i j}^{\prime} \\ \varepsilon_{e}^{2}=\frac{2}{3} \varepsilon_{i j}^{\prime} \varepsilon_{i j}^{\prime}, & \chi_{e}^{2}=\frac{2}{3} \chi_{i j}^{\prime} \chi_{i j}^{\prime}\end{cases}
$$

For the purpose of some specific calculations, the hardening relationship between $\Sigma_{e}$ and $E_{e}$ can be chosen.

The following principle of minimum potential energy may be stated

Consider all admissible displacement fields $u_{i}$ and rotation vector fields $\omega_{i}$ which satisfy $u_{i}=u_{i}^{0}$ and $\omega_{i}=\omega_{i}^{0}$ on the part of the boundaries $S_{u}$ and $S_{\omega}$ respectively. The real displacement fields and the real rotation vector fields render the potential energy $P(\mathbf{u}, \boldsymbol{\omega})$ to be minimum. The potential energy $P(\mathbf{u}, \boldsymbol{\omega})$ is expressed as following,

$$
P(\mathbf{u}, \boldsymbol{\omega})=\int_{V} w(\varepsilon, \chi) \mathrm{d} V-\left(\int_{S_{T}} T_{i}^{0} u_{i} \mathrm{~d} S+\int_{S_{q}} q_{i}^{0} \omega_{i} \mathrm{~d} S\right)
$$

The strain gradient theory proposed by Fleck and Hutchinson (1993) and Fleck et al. (1994) falls within the classification of reduced couple stress theory. The pertinent kinematic quantities in reduced couple stress theory are the displacement $\mathbf{u}$ and the associated material rotation $\theta \equiv(1 / 2)$ curlu. The relative rotation tensor $\alpha$ vanishes for the particular choice $\boldsymbol{\omega} \equiv \boldsymbol{\theta}$ and the curvature tensor $\chi_{i j}=\theta_{i, j}, \chi_{i i}=0$. The force equilibrium and moment equilibrium is the same as Eqs. (6) and (7), respectively, but the moment $\mathbf{m}$ is only the deviatoric part and the spherical part $m_{m}$ can not enter the equilibrium equations.

In this paper, the Cauchy stress is symmetric and the couple stress includes the deviatoric part and the spherical part. The spherical part of couple stress is not zero in the present paper since the independent work-conjugated rotation $\omega$ is introduced and different from other theories. The spherical part of couple stress plays an important role in obtaining the boundary conditions.

Details about the importance of the spherical part's existence can be found in the paper by Green et al. (1968), in which a nonlinear theory of elastic-plastic continua in the presence of simple force and stress dipoles is proposed and used to analyze the problem of torsion of an elastic-plastic cylinder. In their paper, they mentioned that the spherical part of the dipolar stress tensor made no contribution to the equations of motion or to the rate of work equation over any arbitrary closed surface and remained undetermined. In order to provide a determinate theory, they set the spherical part of the dipolar stress tensor to zero at points inside the body, but pointed out that these stresses played an important part in obtaining correct boundary conditions corresponding to the basic differential equations and kept in mind the role played by these stresses in obtaining the boundary conditions. 
The new strain gradient deformation theory reduces to the conventional $J_{2}$ deformation theory in the absence of strain gradient effects, that is, when the geometric length scales are large compared to $l$. In this paper, finite strain effects are neglected: no distinction is made between the initial undeformed configuration and the current deformed configuration.

\section{Comparison with the theory by Shizawa and Zbib (1999)}

Shizawa and Zbib (1999) proposed a thermodynamical theory of gradient elastoplasticity with the microstress conjugate to the dislocation density tensor. In the theory, the dislocation density tensor is an internal variable and the total curvature is not needed. Symmetric Cauchy stress tensor is yielded.

Though the Cauchy stress is symmetric and it is the same as that in the present theory, the theory proposed by Shizawa and Zbib (1999) is only similar to the present theory and not the same one.

(1) In the theory proposed by Shizawa and Zbib (1999), it is the dislocation density tensor not the overall curvature tensor who is taken into account. While the total curvature $\bar{\chi}_{m}$ is taken into account and the dislocation density tensor, the plastic spin tensor and the back stress tensor are set zero, it can recover the conventional Cosserat theory of plasticity and can be regarded as an extension of the theory by Fleck et al. (1994) to the incremental large deformation theory. The present theory in this paper is based on the general couple stress theory. The displacement vector has no relation with the micro rotation vector $\boldsymbol{\omega}$, which is different from that used in Fleck et al. (1994). While the dislocation density tensor, the plastic spin tensor and the back stress tensor are not taken into account in the theory proposed by Shizawa and Zbib, it can not recover the present theory.

(2) The ideas in both theories are similar since the dislocation density tensor can be closely related to the curvature tensor $\chi_{i j}$, that characterizes the Cosserat theory, and both variables give a characteristic length scale of material.

In the theory by Shizawa and Zbib (1999), while

$$
\mathbf{D}^{e}=0, \quad \mathbf{W}^{P}=0, \quad \mathbf{A}=0
$$

The equilibrium equations fit nicely within the mathematical framework of the present theory and the Cauchy stress becomes symmetric, the microstress is similar in role to the overall couple stress in the present paper.

(3) If we introduce the dislocation density tensor $\beta$ into the present theory and it is conjugate to the microstress tensor $v$, the strain energy density function can be expressed

$$
\delta w=\sigma_{e} \delta \varepsilon_{e}+v_{e} \delta \beta_{e}+m_{e} \delta \chi_{e}
$$

where $v_{e}$ is the effective microstress and $\beta_{e}$ denotes the effective dislocation density tensor. 
The scalar deformation measure is represented as

$$
E_{e}^{2}=\varepsilon_{e}^{2}+l_{\beta}^{2} \beta_{e}^{2}+l_{\chi}^{2} \chi_{e}^{2}
$$

where $l_{\beta}$ is also a length scale. Since $l_{\beta}$ and $l_{\chi}$ are both based on the first order of $\boldsymbol{\omega}$, one of them is unnecessary for the purpose of expressing size effects in plasticity. As indicated above, we choose the work-conjugated curvature tensor $\chi_{i j}$ different from that in the theory by Shizawa and Zbib (1999), in which the dislocation density tensor is chosen.

In this case, the work hardening relations are influenced by not only the equivalent curvature but also the equivalent dislocation density; both expressing the effect of the 1st-order strain gradient. This is facially the same as the theory proposed by Shizawa and Zbib (1999) while the total curvature tensor is introduced into their theory (in Section 6.3.2 of the paper written by Shizawa and Zbib, 1999). However, from Eqs. (6.50)-(6.54) in the paper by Shizawa and Zbib (1999) we can find that the couple stress is only the deviatoric part and the Cauchy stress is not symmetric any more, which is the same as the theory proposed by Fleck et al. (1994). In the present theory, though the dislocation density tensor is considered the Cauchy stress is still symmetric and the couple stress consists of the deviatoric and the spherical parts.

\section{Typical analysis}

\subsection{Torsion of thin wires}

A Cartesian coordinate system $\left(x_{1}, x_{2}, x_{3}\right)$ and a cylindrical polar coordinate system $\left(r, \theta, x_{3}\right)$ are introduced as shown in Fig. 1 and $x_{3}$ axis is parallel to the axis of the wire. The radius of the wire is $a . \kappa$ is the twist per unit length of the wire and taken to be positive without loss of generality. Here, this example is dealt as a nonlinear elasticity problem. Take the same displacement field as in classical torsion

$$
u_{1}=-\kappa x_{2} x_{3}, \quad u_{2}=\kappa x_{1} x_{3}, \quad u_{3}=0
$$

The associated non-vanishing components of strain are

$$
\varepsilon_{13}=\varepsilon_{31}=-\frac{1}{2} \kappa x_{2}, \quad \varepsilon_{23}=\varepsilon_{32}=\frac{1}{2} \kappa x_{1}
$$

(a) Using Fleck and Hutchinson's (1993) strain gradient theory, Fleck et al. (1994) have investigated the torsion of thin wires and the components of rotation are

$$
\theta_{1}=-\frac{1}{2} \kappa x_{1}, \quad \theta_{2}=-\frac{1}{2} \kappa x_{2}, \quad \theta_{3}=\kappa x_{3}
$$




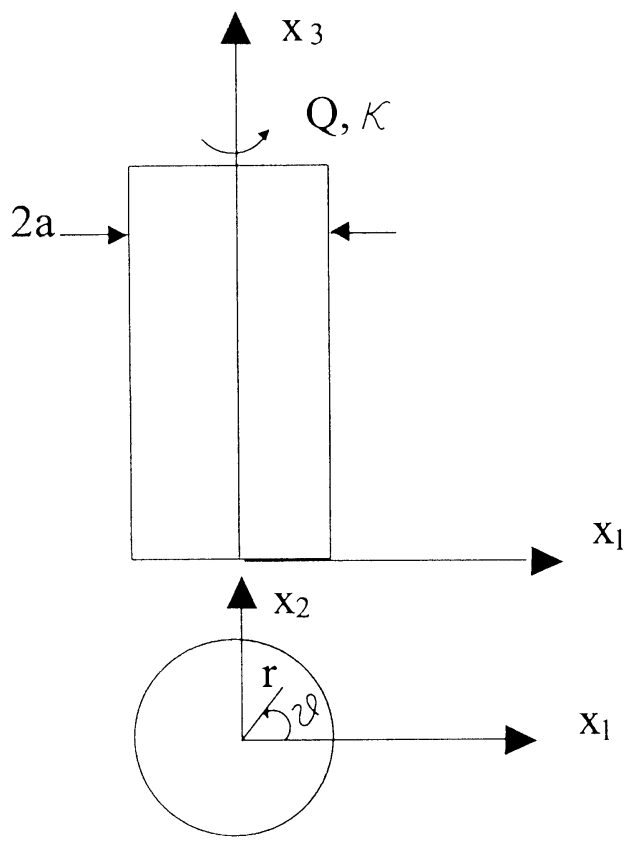

Fig. 1. Coordinate systems $\left(x_{1}, x_{2}, x_{3}\right)$ and $\left(r, \theta, x_{3}\right)$ on a thin wire.

In the cylindrical coordinate system, there are the following components

$$
\theta_{r}=-\frac{1}{2} \kappa r, \quad \theta_{\theta}=0, \quad \theta_{z}=\kappa z
$$

The non-vanishing components of the curvature tensor are

$$
\chi_{11}=\chi_{22}=-\frac{1}{2} \kappa, \quad \chi_{33}=\kappa
$$

Since it is dealt as a nonlinear problem, the elastic strain and the plastic strain need not to be distinguished and the effective strain and strain gradient are

$$
\varepsilon_{e}=\frac{1}{\sqrt{3}} \kappa r, \quad \chi_{e}=\kappa
$$

Fleck et al. (1994) adopted a simple power law relationship between $\Sigma_{e}$ and $E_{e}$

$$
\Sigma_{e}=\Sigma_{0} E_{e}^{n}=\Sigma_{0}\left(\varepsilon_{e}^{2}+l^{2} \chi_{e}^{2}\right)^{n / 2}
$$


The mean stress $\sigma_{k k} / 3$ vanishes. The non-vanishing components of the symmetric part of Cauchy stress and the moment are

$$
\begin{aligned}
& \sigma_{\theta z}=s_{\theta z}=\frac{1}{3} \Sigma_{0} \kappa E_{e}^{n-1} r \\
& m_{r r}=-\frac{1}{3} l^{2} \Sigma_{0} \kappa E_{e}^{n-1}, \quad m_{\theta \theta}=-\frac{1}{3} l^{2} \Sigma_{0} \kappa E_{e}^{n-1}, \quad m_{z z}=\frac{2}{3} l^{2} \Sigma_{0} \kappa E_{e}^{n-1}
\end{aligned}
$$

Combining Eqs. (7) and (36), the non-vanishing anti-symmetric components of Cauchy stress are

$$
\tau_{\theta z}=\frac{1-n}{18} \Sigma_{0} l^{2} \kappa^{3} E_{e}^{n-3} r
$$

The equilibrium equations of force and moment, i.e. Eqs. (6) and (7) are met.

Now, let us check the boundary conditions on the lateral and end faces. First, from the above text, we find that on the lateral face,

$$
\begin{aligned}
& \sigma_{r r}+\tau_{r r}=\sigma_{\theta r}+\tau_{\theta r}=\sigma_{z r}+\tau_{z r}=0 \quad \text { on } \quad r=a \\
& m_{\theta r}=m_{z r}=0 \quad \text { on } \quad r=a \\
& m_{r r}=-\frac{1}{3} l^{2} \Sigma_{0} \kappa^{n}\left(\frac{a^{2}}{3}+l^{2}\right)^{\frac{n-1}{2}} \quad \text { on } \quad r=a
\end{aligned}
$$

From Eq. (40), we find that the couple stress $m_{r r}$ on the lateral face is not equal to zero. In fact, $m_{r r}$ here is only the deviatoric part, the spherical part must be added to meet the boundary conditions but Koiter (1964) has proved that in the reduced couple stress theory the spherical part of the couple stress does not enter the field equations or the constitutive equations. The spherical part can not be determined.

On the end faces there are the following boundary conditions,

$$
\begin{aligned}
& u_{r}=0, \quad u_{\theta}=0, \quad u_{z}=0 \quad \text { on } \quad z=0 \\
& u_{r}=0, \quad u_{\theta}=\kappa r L, \quad u_{z}=0 \quad \text { on } \quad z=L \\
& \theta_{r}=-\frac{1}{2} \kappa r, \quad \theta_{\theta}=0, \quad \theta_{z}=0 \quad \text { on } \quad z=0 \\
& \theta_{r}=-\frac{1}{2} \kappa r, \quad \theta_{\theta}=0, \quad \theta_{z}=\kappa L \quad \text { on } \quad z=L
\end{aligned}
$$

where $L$ is the total length of the thin wire as shown in Fig. 1. 
(b) Now, we use the new strain gradient theory to investigate the same problem. In the cylindrical coordinate system, the equilibrium equations of stress and couple stress are expressed as follows,

$$
\begin{aligned}
& \left\{\begin{array}{l}
\frac{\partial \sigma_{r r}}{\partial r}+\frac{1}{r} \frac{\partial \sigma_{r \theta}}{\partial \theta}+\frac{\partial \sigma_{r z}}{\partial z}+\frac{\sigma_{r r}-\sigma_{\theta \theta}}{r}=0 \\
\frac{\partial \sigma_{\theta r}}{\partial r}+\frac{1}{r} \frac{\partial \sigma_{\theta \theta}}{\partial \theta}+\frac{\partial \sigma_{\theta z}}{\partial z}+\frac{\sigma_{\theta r}+\sigma_{r \theta}}{r}=0 \\
\frac{\partial \sigma_{z r}}{\partial r}+\frac{1}{r} \frac{\partial \sigma_{z \theta}}{\partial \theta}+\frac{\partial \sigma_{z z}}{\partial z}+\frac{\sigma_{z r}}{r}=0
\end{array}\right. \\
& \left\{\begin{array}{l}
\frac{\partial m_{r r}}{\partial r}+\frac{1}{r} \frac{\partial m_{r \theta}}{\partial \theta}+\frac{\partial m_{r z}}{\partial z}+\frac{m_{r r}-m_{\theta \theta}}{r}=0 \\
\frac{\partial m_{\theta r}}{\partial r}+\frac{1}{r} \frac{\partial m_{\theta \theta}}{\partial \theta}+\frac{\partial m_{\theta z}}{\partial z}+\frac{m_{\theta r}+m_{r \theta}}{r}=0 \\
\frac{\partial m_{z r}}{\partial r}+\frac{1}{r} \frac{\partial m_{z \theta}}{\partial \theta}+\frac{\partial m_{z z}}{\partial z}+\frac{m_{z r}}{r}=0
\end{array}\right.
\end{aligned}
$$

In order to compare the results of both strain gradient theories reasonably, the hardening relation, the traction boundary condition and the displacement boundary condition are taken to be the same as that used in Fleck et al. (1994). The solid is assumed to be incompressible for both strain and curvature tensors. The boundary conditions for couple stress on the lateral face can be expressed as follows,

$$
\begin{aligned}
& m_{\theta r}=m_{z r}=0 \text { on } r=a \\
& m_{r r}=-\frac{1}{3} l^{2} \Sigma_{0} \kappa^{n}\left(\frac{a^{2}}{3}+l^{2}\right)^{\frac{n-1}{2}} \text { on } r=a
\end{aligned}
$$

Of course, the boundary condition (48a) is the same as Eq. (40) but it is not reasonable. An alternative realistic boundary condition is

$$
m_{r r}=0 \text { on } r=a
$$

We assume that there exists an ultra-tin homogeneous layer on the surface $S_{\omega}$, then we have the same rotation boundary conditions as that in Fleck et al. (1994), that is

$$
\omega_{i}=\omega_{i}^{0}=\theta_{i}^{0} \text { on } S_{\omega}
$$


Then the rotation boundary conditions on the end faces are

$$
\begin{aligned}
& \omega_{r}=-\frac{1}{2} \kappa r, \quad \omega_{\theta}=0, \quad \omega_{z}=0 \text { on } z=0 \\
& \omega_{r}=-\frac{1}{2} \kappa r, \quad \omega_{\theta}=0, \quad \omega_{z}=\kappa L \text { on } z=L
\end{aligned}
$$

We can find that the above boundary value problem has the following solutions:

$$
\begin{aligned}
& u_{1}=-\kappa x_{2} x_{3}, \quad u_{2}=\kappa x_{1} x_{3}, \quad u_{3}=0 \\
& \omega_{r}=-\frac{1}{2} \kappa r, \quad \omega_{\theta}=0, \quad \omega_{z}=\kappa z
\end{aligned}
$$

It is verified as the following.

From the displacement field, we have the non-vanishing components of strain

$$
\varepsilon_{13}^{\prime}=\varepsilon_{31}^{\prime}=-\frac{1}{2} \kappa x_{2}, \quad \varepsilon_{23}^{\prime}=\varepsilon_{32}^{\prime}=\frac{1}{2} \kappa x_{1}, \quad \varepsilon_{e}=\frac{1}{\sqrt{3}} \kappa r
$$

and the non-vanishing deviatoric components of curvature are

$$
\chi_{11}^{\prime}=\chi_{22}^{\prime}=-\frac{1}{2} \kappa, \quad \chi_{33}^{\prime}=\kappa, \quad \chi_{e}=\kappa
$$

The spherical part of curvature is

$$
\chi_{m}=\frac{1}{3} \chi_{k k}=0
$$

Here, the simple power law relationship between $\Sigma_{e}$ and $E_{e}$, Eq. (34) is adopted also, the non-vanishing component of Cauchy stress only is

$$
\sigma_{\theta z}=s_{\theta z}=\frac{1}{3} \Sigma_{0} \kappa E_{e}^{n-1} r
$$

The non-vanishing deviatoric components of couple stress are

$$
m_{r r}^{\prime}=-\frac{1}{3} l^{2} \Sigma_{0} \kappa E_{e}^{n-1}, \quad m_{\theta \theta}^{\prime}=-\frac{1}{3} l^{2} \Sigma_{0} \kappa E_{e}^{n-1}, \quad m_{z z}^{\prime}=\frac{2}{3} l^{2} \Sigma_{0} \kappa E_{e}^{n-1}
$$


From Eq. (57), we find that the non-vanishing component of Cauchy stress is only related with $r$, the equilibrium equations of stress, i.e. Eq. (45) are met.

Since the components of couple stress in Eq. (58) are only related with $r$, Eq. (46) can be reduced

$$
\left\{\begin{array}{l}
\frac{\partial m_{r r}}{\partial r}+\frac{m_{r r}-m_{\theta \theta}}{r}=0 \\
\frac{1}{r} \frac{\partial m_{m}}{\partial \theta}=0 \\
\frac{\partial m_{m}}{\partial z}=0
\end{array}\right.
$$

where $m_{i j}=m_{i j}^{\prime}+m_{m} \delta_{i j}$ and $m_{m}$ denotes the spherical part of couple stress.

Now we introduce $K_{1}$, which is called the volumetric module of bend-torsion, then we have

$$
m_{m}=K_{1} l^{2} \chi_{m}
$$

Since the solid is assumed to be incompressible for bend-torsion, thus $K_{1} \rightarrow \infty$. $m_{m}$ can not be determined from Eq. (60), and becomes an independent unknown variable.

From Eq. (59) one finds

$$
m_{m}=A-m_{r r}^{\prime}
$$

where $A$ is a constant to be determined by the boundary conditions. From Eq. (48a) it follows that $m_{m}(r=a)=0$, then we have

$$
m_{m}=\frac{1}{3} l^{2} \kappa^{n} \Sigma_{0}\left[\left(\frac{1}{3} r^{2}+l^{2}\right)^{\frac{n-1}{2}}-\left(\frac{1}{3} a^{2}+l^{2}\right)^{\frac{n-1}{2}}\right]
$$

The stress and couple stress tractions on the end face for the new strain gradient theory are Eq. (62) and the following,

$$
\begin{aligned}
& m_{r z}^{\prime}=m_{\theta z}^{\prime}=0, \quad m_{z z}^{\prime}=\frac{2}{3} l^{2} \Sigma_{0} \kappa E_{e}^{n-1} \\
& \sigma_{r z}=\sigma_{z z}=0, \quad \sigma_{\theta z}=s_{\theta z}=\frac{1}{3} \Sigma_{0} \kappa E_{e}^{n-1} r
\end{aligned}
$$


Here, the torque produced on the end face for both strain gradient theories is investigated. In Fleck et al. (1994), the torque produced by the anti-symmetrical part of Cauchy stress on the end face is

$$
\begin{aligned}
Q_{1} & =\int_{0}^{2 \pi} \int_{0}^{a} \tau_{\theta z} r^{2} \mathrm{~d} r \mathrm{~d} \theta \\
& =\frac{2 \pi l^{2} \Sigma_{0} \kappa^{n}}{n+1}\left[\left(\frac{a^{2}}{3}+l^{2}\right)^{\frac{n+1}{2}}-l^{n+1}\right]-\frac{\pi l^{2} \Sigma_{0} \kappa^{n} a^{2}}{3}\left(\frac{a^{2}}{3}+l^{2}\right)^{\frac{n-1}{2}}
\end{aligned}
$$

The torque produced by the spherical part of moment in the new strain gradient theory is

$$
\begin{aligned}
Q_{2} & =\int_{0}^{2 \pi} \int_{0}^{a} m_{m} r \mathrm{~d} r \mathrm{~d} \theta \\
& =\frac{2 \pi l^{2} \Sigma_{0} \kappa^{n}}{n+1}\left[\left(\frac{a^{2}}{3}+l^{2}\right)^{\frac{n+1}{2}}-l^{n+1}\right]-\frac{\pi l^{2} \Sigma_{0} \kappa^{n} a^{2}}{3}\left(\frac{a^{2}}{3}+l^{2}\right)^{\frac{n-1}{2}}
\end{aligned}
$$

It is clear that $Q_{1}=Q_{2}$.

The overall torque on the end face for both strain gradient theories is same,

$$
\begin{aligned}
Q & =\int_{0}^{2 \pi} \int_{0}^{a} s_{\theta z} r^{2} \mathrm{~d} r \mathrm{~d} \theta+\int_{0}^{2 \pi} \int_{0}^{a} m_{z z}^{\prime} r \mathrm{~d} r \mathrm{~d} \theta+Q_{1} \\
& =\frac{6 \pi \Sigma_{0} \kappa^{n}}{n+3}\left[\left(\frac{a^{2}}{3}+l^{2}\right)^{\frac{n+3}{2}}-l^{n+3}\right]-\frac{\pi \Sigma_{0} \kappa^{n} l^{2} a^{2}}{3}\left(\frac{a^{2}}{3}+l^{2}\right)^{\frac{n-1}{2}}
\end{aligned}
$$

If we let the moments on the lateral face to be zero exactly, that is Eq. (48b), we obtain,

$$
m_{m}=\frac{1}{3} l^{2} \kappa^{n} \Sigma_{0}\left(\frac{1}{3} r^{2}+l^{2}\right)^{\frac{n-1}{2}}
$$

The torque produced by the spherical part of moment in the new strain gradient theory is

$$
Q_{1}=\int_{0}^{2 \pi} \int_{0}^{a} m_{m} r \mathrm{~d} r \mathrm{~d} \theta=\frac{2 \pi l^{2} \Sigma_{0} \kappa^{n}}{n+1}\left[\left(\frac{a^{2}}{3}+l^{2}\right)^{\frac{n+1}{2}}-l^{n+1}\right]
$$


The overall torque produced by $\sigma_{\theta z}, m_{z z}^{\prime}, m_{m}$ on the end face is

$$
Q=\frac{6 \pi \Sigma_{0} \kappa^{n}}{n+3}\left[\left(\frac{a^{2}}{3}+l^{2}\right)^{\frac{n+3}{2}}-l^{n+3}\right]
$$

The above equation is the same as Eq. (6.9) in Fleck et al. (1994).

(c) In this section, $K_{1}$ is taken to be a finite value and the same problem is solved as follows.

The displacement field is taken as Eq. (28) and the boundary conditions for couple stress on the lateral face are expressed as

$$
m_{r r}=m_{\theta r}=m_{z r}=0 \text { on } r=a
$$

The boundary conditions on the end faces are taken as

$$
\left\{\begin{array}{llll}
\omega_{z}=0, & \omega_{\theta}=0 & & z=0 \\
\omega_{z}=\kappa L, & \omega_{\theta}=0 & & \\
& \text { on } & & \\
& & &
\end{array}\right.
$$

We assume that $\chi_{i j}$ has only relation with $r$, then one can obtain the following solutions for the boundary value problem,

$$
\begin{aligned}
& \omega_{r}=-\frac{1}{2} \kappa r+\tilde{\omega}_{r}(r), \quad \omega_{\theta}=0, \quad \omega_{z}=\kappa z \\
& \left\{\begin{array}{l}
m_{r r}^{\prime}=\frac{2}{3} \Sigma_{0} E_{e}^{n-1} l^{2}\left(-\frac{\kappa}{2}+\frac{2}{3} \tilde{\omega}_{r}^{\prime}-\frac{1}{3} \frac{\tilde{\omega}_{r}}{r}\right) \\
m_{\theta \theta}^{\prime}=\frac{2}{3} \Sigma_{0} E_{e}^{n-1} l^{2}\left(-\frac{\kappa}{2}+\frac{2}{3} \frac{\tilde{\omega}_{r}}{r}-\frac{1}{3} \tilde{\omega}_{r}^{\prime}\right) \\
m_{z z}^{\prime}=\frac{2}{3} \Sigma_{0} E_{e}^{n-1} l^{2}\left(\kappa-\frac{1}{3} \tilde{\omega}_{r}^{\prime}-\frac{1}{3} \frac{\tilde{\omega}_{r}}{r}\right) \\
m_{m}=\frac{K_{1} l^{2}}{3}\left(\tilde{\omega}_{r}^{\prime}+\frac{\tilde{\omega}_{r}}{r}\right)
\end{array}\right.
\end{aligned}
$$

and $\tilde{\omega}_{r}$ can be obtained from the first equation of Eq. (59)

$$
\begin{aligned}
& 2(n-1) E_{e}^{n-2}\left(-\frac{\kappa}{2}+\frac{2}{3} \tilde{\omega}_{r}^{\prime}-\frac{1}{3} \frac{\tilde{\omega}_{r}}{r}\right) E_{e}^{\prime}+2 E_{e}^{n-1}\left(\frac{2}{3} \tilde{\omega}_{r}^{\prime \prime}-\frac{1}{3} \frac{\tilde{\omega}_{r}^{\prime}}{r}+\frac{1}{3} \frac{\tilde{\omega}_{r}}{r^{2}}\right) \\
& \quad+2 E_{e}^{n-1}\left(\tilde{\omega}_{r}^{\prime} r^{-1}-\tilde{\omega}_{r} r^{-2}\right)+\frac{K_{1}}{\Sigma_{0}}\left(\tilde{\omega}_{r}^{\prime \prime}+\tilde{\omega}_{r}^{\prime} r^{-1}-\tilde{\omega}_{r} r^{-2}\right) \\
& \quad=0
\end{aligned}
$$


where

$$
\begin{aligned}
E_{e}^{2}= & \frac{\kappa^{2} r^{2}}{3}+\frac{2}{3} l^{2}\left[\left(-\frac{\kappa}{2}+\frac{2}{3}{\tilde{\omega^{\prime}}}_{r}-\frac{1}{3} \frac{\tilde{\omega}_{r}}{r}\right)^{2}\right. \\
& \left.+\left(-\frac{\kappa}{2}+\frac{2}{3} \frac{\tilde{\omega}_{r}}{r}-\frac{1}{3} \tilde{\omega}_{r}^{\prime}\right)^{2}+\left(\kappa-\frac{1}{3} \tilde{\omega}_{r}^{\prime}-\frac{1}{3} \frac{\tilde{\omega}_{r}}{r}\right)^{2}\right]
\end{aligned}
$$

The boundary conditions for Eq. (75) are

$$
\tilde{\omega}_{r}(r=0)=0, \quad m_{r r}(r=a)=0
$$

Combining Eqs. (75) and (77) and through numerical calculation we can obtain $\tilde{\omega}_{r}$. For a set of parameters, $K_{1} / \Sigma_{0}=1, n=0.5, l=2.82 \mu \mathrm{m}, a=1 \mu \mathrm{m}, \kappa=0.1$, the relation curves $r \sim \tilde{\omega}_{r}$ and $r \sim m_{r r}$ are shown in Fig. 3 and 4.

\subsection{Ultra-thin beam bend}

Stolken and Evans (1988) did the bend experiment and observed a strong effect whereby thin beams display much stronger plastic work hardening than thick ones and no size dependence is observed in the tension test.

Cartesian $\left(x_{1}, x_{2}, x_{3}\right)$ coordinates are adopted as shown in Fig. $2 . \kappa$ is the curvature, $h$ is the beam's thickness and $b$ is the beam's width. Also, this example is dealt with as a nonlinear problem.

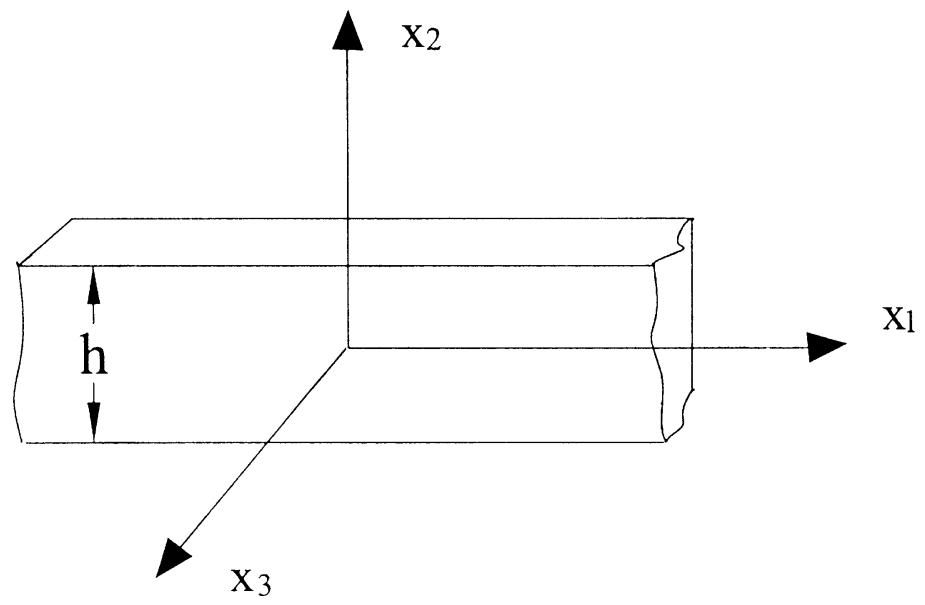

Fig. 2. Coordinate system $\left(x_{1}, x_{2}, x_{3}\right)$ on an ultra-thin beam. 
(a) Stolken and Evans (1998) gave the solution based on the Fleck-Hutchinson's strain gradient theory, the displacements are

$$
u_{1}=\kappa x_{1} x_{2}, \quad u_{2}=-\kappa\left(x_{1}^{2}+x_{2}^{2}\right) / 2, \quad u_{3}=0
$$

The non-vanishing strain components are

$$
\varepsilon_{11}=-\varepsilon_{22}=\kappa x_{2}
$$

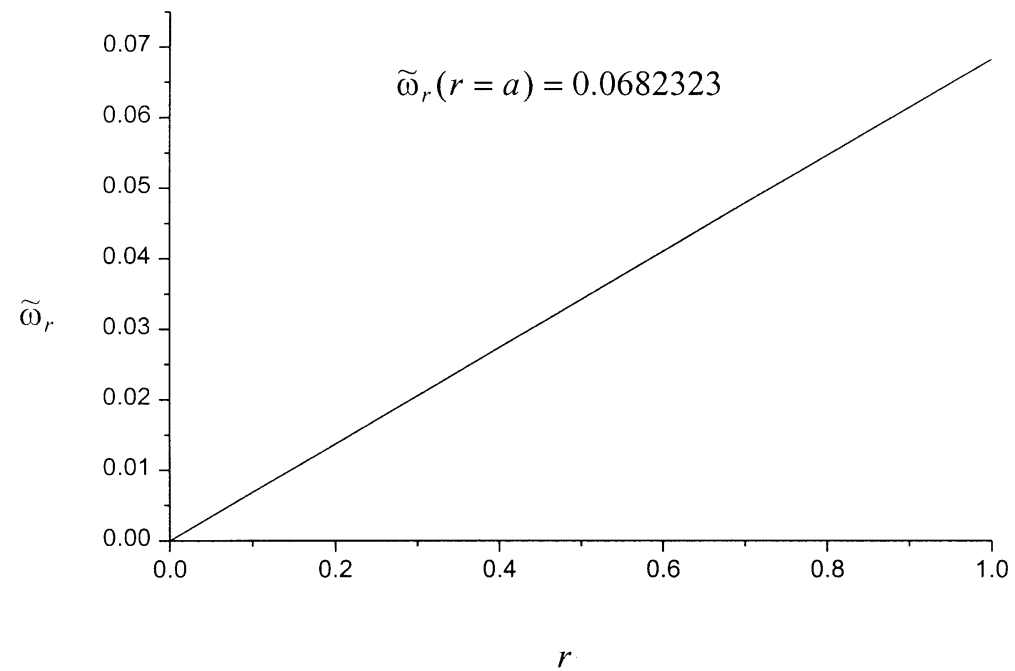

Fig. 3. Relation curve of $r \sim \tilde{\omega}_{r}$.

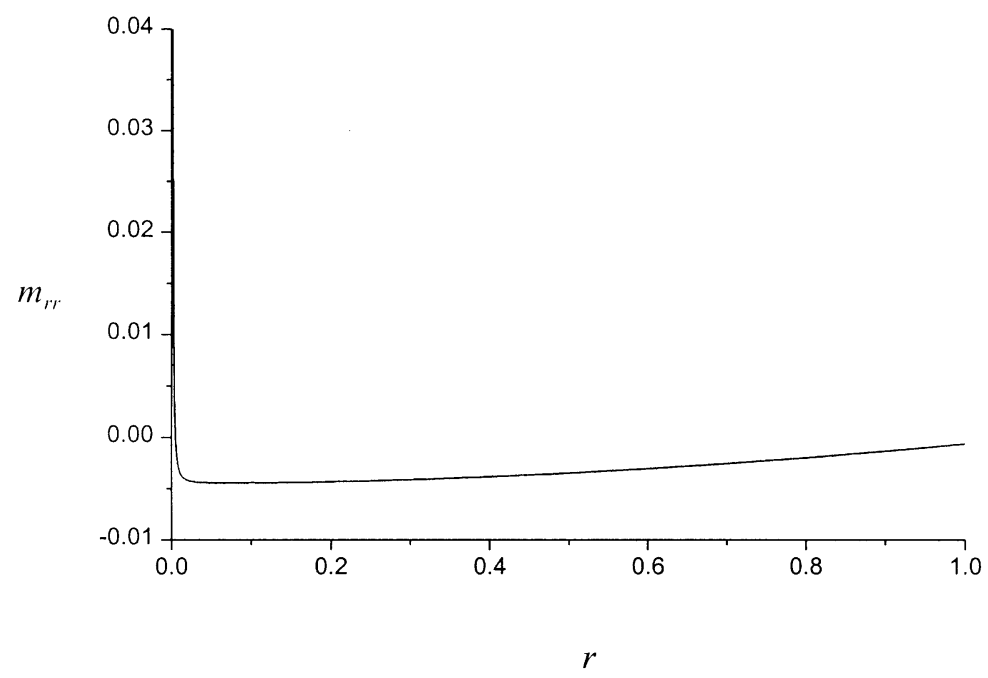

Fig. 4. Relation curve of $r \sim m_{r r}$. 
The components of the associated rotation are

$$
\theta_{1}=0, \quad \theta_{2}=0, \quad \theta_{3}=-\kappa x_{1}
$$

The non-vanishing components of curvature tensor are

$$
\chi_{31}=-\kappa
$$

The effective strain and the effective curvature tensor are

$$
\varepsilon_{e}=\frac{2}{\sqrt{3}} \kappa\left|x_{2}\right|, \quad \chi_{e}=\sqrt{\frac{2}{3}} \kappa
$$

From simulating the tensile test results of thin beams, the relation between the stress and plastic strain can be expressed as (Stolken and Evans, 1998)

$$
\sigma=\Sigma_{0}+\varepsilon_{p l} E_{p}
$$

where $\Sigma_{0}$ is the yield strength, $\varepsilon_{p l}$ is the plastic strain and $E_{p}$ the hardening coefficient. Then the relation between the effective stress and effective strain can be obtained

$$
\sigma_{e}=\frac{\sqrt{3}}{2} \Sigma_{0}+\frac{3}{4} E_{p} \varepsilon_{e p}
$$

where $\varepsilon_{e p}$ is the effective plastic strain.

The hardening relation including the effect of strain gradient plasticity is

$$
\Sigma_{e}=\frac{\sqrt{3}}{2} \Sigma_{0}+\frac{3}{4} E_{p} E_{e}, \quad E_{e}=\sqrt{\varepsilon_{e}^{2}+l^{2} \chi_{e}^{2}}
$$

The non-vanishing deviatoric components of the symmetric part of Cauchy stress and the moment are

$$
s_{11}=\frac{2 \Sigma_{e}}{3 E_{e}} \kappa x_{2}, \quad s_{22}=-\frac{2 \Sigma_{e}}{3 E_{e}} \kappa x_{2}, \quad m_{31}=-\frac{2 \Sigma_{e}}{3 E_{e}} l^{2} \kappa
$$

Then

$$
\begin{aligned}
& \sigma_{11}=\frac{4 \Sigma_{e}}{3 E_{e}} \kappa x_{2}, \quad \sigma_{33}=\frac{2 \Sigma_{e}}{3 E_{e}} \kappa x_{2} \\
& \tau_{i j}=0, \quad(i, j=1,2,3)
\end{aligned}
$$

The equilibrium equations of force and moment, i.e. Eqs. (6) and (7) are all met. 
Let us check the boundary conditions on the lateral faces,

$$
\begin{aligned}
& t_{12}=t_{22}=t_{32}=0, \quad m_{12}=m_{22}=m_{32}=0, \quad \text { on } x_{2}= \pm h / 2 \\
& t_{13}=t_{23}=0, \quad m_{13}=m_{23}=m_{33}=0 \text { on } x_{3}= \pm b / 2 \\
& t_{33}=\frac{2 \Sigma_{e}}{3 E_{e}} \kappa x_{2} \text { on } x_{3}= \pm b / 2
\end{aligned}
$$

The boundary conditions on the end faces are

$$
\begin{aligned}
& t_{11}=\frac{4 \Sigma_{e}}{3 E_{e}} \kappa x_{2}, \quad t_{21}=t_{31}=0 \text { on } x_{1}= \pm L / 2 \\
& m_{11}=m_{21}=0, \quad m_{31}=-\frac{2 \Sigma_{e}}{3 E_{e}} l^{2} \kappa \text { on } x_{1}= \pm L / 2
\end{aligned}
$$

(b) Now, we use the new strain gradient theory to investigate the same problem. Similar to the problem of thin wire torsion, in order to compare both strain gradient theories, the hardening relation, the traction boundary conditions for stress and couple stress are taken to be the same as that used in Stolken and Evans (1998). The solid is assumed to be incompressible for strain tensor.

We can find that the solutions to this boundary value problem are as follows,

$$
\begin{aligned}
& u_{1}=\kappa x_{1} x_{2}, \quad u_{2}=-\kappa\left(x_{1}^{2}+x_{2}^{2}\right) / 2, \quad u_{3}=0 \\
& \omega_{1}=0, \quad \omega_{2}=0, \quad \omega_{3}=-\kappa x_{1}
\end{aligned}
$$

It can be verified easily that the equilibrium equations of both stress and couple stress for the new strain gradient theory are all met.

\section{The $J_{2}$ flow theory version}

In this section, the strain gradient $J_{2}$ flow theory version is given briefly by following the new strain gradient deformation theory in Section 3.

The strain tensor consists of elastic and plastic parts. The curvature tensor also consists of the two parts. Thus, the strain rate and the curvature rate can be expressed,

$$
\dot{\varepsilon}_{i j}=\dot{\varepsilon}_{i j}^{e l}+\dot{\varepsilon}_{i j}^{p l} \quad \dot{\chi}_{i j}=\dot{\chi}_{i j}^{e l}+\dot{\chi}_{i j}^{p l}
$$

Assuming the existence of couple stresses in the elastic-plastic body. The elastic strain state $\left(\varepsilon_{i j}^{e l}, \chi_{i j}^{e l}\right)$ is obtained

$$
\varepsilon_{i j}^{e l}=\mu_{i j k l} \sigma_{k l} \quad l_{e l} \chi_{i j}^{e l}=M_{i j k l} l_{e l}^{-1} m_{k l}
$$


where $\mu_{i j k l}$ and $M_{i j k l}$ are the flexible tensors and $l_{e l}$ is introduced in order to partition the curvature tensor $\chi$ into its elastic part and plastic part.

The magnitude of the elastic couple stresses may be made arbitrarily small by choosing the ratio $l_{e l} / l$ to be sufficiently small, where $l$ is the length scale corresponding to the plastic couple stresses. The plastic strain $\varepsilon_{i j}^{p l}$ and the plastic curvature $\chi_{i j}^{p l}$ are determined by integration of $\dot{\varepsilon}_{i j}^{p l}$ and $\dot{\chi}_{i j}^{p l}$ with respect to time respectively. The yield surface $\Phi$ is written as

$$
\Phi=\Phi\left(\sigma_{e}, m_{e}, Y\right)
$$

where $\sigma_{e}=\sqrt{\frac{3}{2} s_{i j} s_{i j}}$ is the Von Mises effective stress, $m_{e}=\sqrt{\frac{3}{2} m_{i j}^{\prime} m_{i j}^{\prime}}$ is the effective couple stress, and $Y$ is the current flow stress.

Plastic flow is normal to the yield surface such that

$$
\dot{\varepsilon}_{i j}^{p l}=\lambda \frac{\partial \Phi}{\partial \sigma_{i j}} \quad l \dot{\chi}_{i j}^{p l}=l^{-1} \lambda \frac{\partial \Phi}{\partial m_{i j}}
$$

According to Fleck and Hutchinson (1993), the single scalar measure $\Sigma_{e}^{2}=\sigma_{e}^{2}+$ $l^{-2} m_{e}^{2}$ is called the overall effective stress and the overall effective plastic strain is defined as $\dot{E}_{e}^{p l}=\sqrt{\left(\dot{\varepsilon}_{e}^{p l}\right)^{2}+\left(l \dot{\chi}_{e}^{p l}\right)^{2}}$, where the effective strain rate is $\dot{\varepsilon}_{e}^{p l}=\sqrt{\frac{2}{3} \dot{\varepsilon}_{i j}^{p l} \dot{\varepsilon}_{i j}^{p l}}$ and the effective curvature rate is $\dot{\chi}_{e}^{p l}=\sqrt{\frac{2}{3} \dot{\chi}_{i j}^{p l} \dot{\chi}_{i j}^{p l}}$. Then the yield surface (98) generalizes to

$$
\Phi\left(\Sigma_{e}, \quad Y\right)=\Sigma_{e}-Y=0
$$

The plastic strain rate and the plastic curvature rate can be given

$$
\dot{\varepsilon}_{i j}^{p l}=\frac{3}{2 h\left(\Sigma_{e}\right)} \frac{s_{i j}}{\Sigma_{e}} \dot{\Sigma}_{e} \quad l \dot{\chi}_{i j}^{p l}=\frac{3}{2 h\left(\Sigma_{e}\right)} \frac{l^{-1} m_{i j}^{\prime}}{\Sigma_{e}} \dot{\Sigma}_{e}
$$

where

$$
\dot{\Sigma}_{e}=\frac{3}{2} \frac{s_{i j}}{\Sigma_{e}} \dot{s}_{i j}+\frac{3}{2} \frac{l^{-1} m_{i j}^{\prime}}{\Sigma_{e}} l^{-1} \dot{m}_{i j}^{\prime}
$$

and $h$ equals the tangent modulus of the stress $\Sigma_{e}$ versus plastic strain curve in simple tension, i.e. $h=\dot{\Sigma}_{e} / \dot{E}_{e}^{p l}$.

\section{Conclusions}

(1) The present theory fits within the mathematical framework of the general couple stress theory, but the present deformation theory differs from all existing strain gradient theories. 
(2) In the new strain gradient deformation theory, the micro-rotation $\omega_{i}$ is different from the material rotation $\theta_{i}$, generally speaking that $\alpha_{i j} \neq 0$, i.e. the particles' own relative rotation with respect to the material is considered. In the strain energy function, it is assumed that $\alpha_{i j}$ has no contribution to the strain energy density, which renders $\tau_{i j} \equiv 0$ and symmetric Cauchy stress tensor.

(3) Since $\omega_{i} \neq \theta_{i}, \chi_{k k} \neq 0$, the volumetric modulus of bend-torsion $K_{1}$ can be naturally introduced.

(4) Using the new strain gradient deformation theory, the moment on the free boundary can be met exactly and the spherical part of the moment can be obtained.

(5) The micro-rotation, $\omega_{i}$, is treated as an independent kinematic quantity with no direct dependence upon $u_{i}$, this enables the use of $C_{0}$ continuous elements in a finite element formulation, which will give great benefit to the finite element calculation to avoid dramatic sensitive to the element formulation.

\section{Acknowledgements}

This work is supported by the National Natural Science Foundation of China (No. 19704100), National Science Foundation of Chinese Academy of Sciences (Project KJ951-1-20), CAS K. C. Wong Post-doctoral Research Award Fund and the Post-doctoral Science Fund of China.

\section{Appendix}

In the new strain gradient theory, $\omega_{i}$ is the work conjugate rotation, which is different from $\theta_{i}$. The following discussion will give an explanation of the physical meaning of the work conjugate rotation $\omega_{i}$.

Consider a unit cell subject only to a prescribed traction of couple stress $q_{i}^{0}$ on the surface,

$$
\begin{aligned}
& q_{i}=q_{i}^{0}=\bar{m}_{i j} n_{j} \quad \text { on } S \\
& T_{i}=T_{i}^{0}=0 \quad \text { on } S
\end{aligned}
$$

where $\bar{m}_{i j}$ is constant overall couple stress.

The work done by the couple stress on the virtual curvature $\delta \chi_{i j}$ can be expressed as

$$
\delta w=\int_{V} m_{i j} \delta \chi_{i j} \mathrm{~d} V
$$

If the material inside the unit cell is inhomogeneous or the unit cell contains some microstructures, then we can suppose that the virtual displacements take the form

$$
\delta u_{i}=\delta u_{i}^{(0)}+\delta \tilde{u}_{i} \quad \mathbf{x} \in V
$$


where

$$
\begin{array}{ll}
\delta u_{i}^{(0)}=\frac{1}{2} \delta \bar{\eta}_{j k i} x_{j} x_{k} \quad \mathbf{x} \in S \\
\delta \tilde{u}_{i}=0 \quad \text { on } S
\end{array}
$$

and $\bar{\eta}_{j k i}$ denotes the constant second gradient of the displacement. Then the virtual curvature tensor is

$$
\delta \chi_{i j}=\delta \theta_{i, j}=\delta \theta_{i, j}^{(0)}+\delta \tilde{\theta}_{i, j}=\delta \bar{\chi}_{i j}+\delta \tilde{\chi}_{i j}
$$

where

$$
\begin{aligned}
& \delta \bar{\chi}_{i j}=\frac{1}{2} e_{i l k} \delta \bar{\eta}_{l j k} \\
& \delta \tilde{\chi}_{i j}=\delta \tilde{\theta}_{i, j}
\end{aligned}
$$

Substituting the above equation into Eq. (A3), one can obtain

$$
\begin{aligned}
\int_{V} m_{i j} \delta \chi_{i j} \mathrm{~d} V & =V \bar{m}_{i j} \delta \bar{\chi}_{i j}+\int_{S} m_{i j} n_{j} \delta \tilde{\theta}_{i} \mathrm{~d} S \\
& =V \bar{m}_{i j} \delta \bar{\chi}_{i j}+\bar{m}_{i j} \int_{S} n_{j} \delta \tilde{\theta}_{i} \mathrm{~d} S \\
& =V \bar{m}_{i j} \delta \bar{\chi}_{i j}+\bar{m}_{i j} \cdot \int_{V} \delta \tilde{\chi}_{i j} \mathrm{~d} V \\
& =V \bar{m}_{i j}\left(\delta \bar{\chi}_{i j}+\delta \bar{\rho}_{i j}\right) \\
& =V \bar{m}_{i j} \delta \bar{\varpi}_{i j}
\end{aligned}
$$

where

$$
\delta \bar{\rho}_{i j}=\frac{1}{V} \int_{S} n_{j} \delta \tilde{\theta}_{i} \mathrm{~d} S=\frac{1}{V} \int_{V} \delta \tilde{\chi}_{i j} \mathrm{~d} V
$$

and $\bar{\varpi}_{i j}=\bar{\chi}_{i j}+\bar{\rho}_{i j}$

Here, $\bar{\varpi}_{i j}$ is called the overall curvature tensor.

Generally speaking $\left.\delta \tilde{\theta}_{i}\right|_{S} \neq 0$, hence $\delta \bar{\rho}_{i j}$ does not vanish. Obviously, $\bar{\varpi}_{i j}$ is the work conjugate of $\bar{m}_{i j}$. 


\section{References}

Acharya, A., Bassani, J.L., 1995. On non-local flow theories that preserve the classical structure of incremental boundary value problems. In: Micromechanics of Plasticity and Damage of Multiphase Materials, IUTAM Symposium, Paris, 29 August to 1 September.

Aifantis, E.C., 1984. On the microstructural origin of certain inelastic models. Trans. ASME J. Eng. Mater. Technol. 106, 326.

Aifantis, E.C., 1987. The physics of plastic deformation. Int. J. Plast. 3, 211.

Chen, S.H., Wang, T.C., 2000. A new hardening law for strain gradient plasticity. Acta Mater. 48 (16), 3997.

Cosserat, E., Cosserat, F., 1909. Theorie des Corps Deformables. Herman et fils, Paris.

Dillon, O.W., Kratochvil, J.R., Kratochvil, J., 1970. A strain gradient theory of plasticity. Int. J. Solids Struct. 6, 1513.

Elssner, G., Korn, D., Ruehle, M., 1994. The influence of interface impurities on fracture energy of UHV diffusion bonded metal-ceramic bicrystals. Scr. Metall. Mater. 31, 1037.

Eringen, A.C., 1968. Theory of micropolar elasticity. In: Leibowitz, H. (Ed.), Fracture, An Advanced Treatise. Academic, New York, p. 621.

Fleck, N.A., Hutchinson, J.W., 1993. A phenomenological theory for strain gradient effects in plasticity. J. Mech. Phys. Solids 41, 1825.

Fleck, N.A., Hutchinson, J.W., 1997. In: Hutchinson, J.W., Wu, T.Y. (Eds.), Strain Gradient Plasticity. Advances in Applied Mechanics, Vol. 33. Academic, New York, p. 295.

Fleck, N.A., Muller, G.M., Ashby, M.F., Hutchinson, J.W., 1994. Strain gradient plasticity: theory and experiment. Acta Metall. Mater. 42, 475.

Gao, H., Huang, Y., Nix, W.D., Hutchinson, J.W., 1999. Mechanism-based strain gradient plasticity-I. Theory. J. Mech. Phys. Solids 47, 1239.

Green, A.E., Mcinnis, B.C., Naghdi, P.M., 1968. Elastic-plastic continua with simple force dipole. Int. J. Eng. Sci. 6, 373.

Hutchinson, J.W., 1997. Linking scales in mechanics. In: Karihaloo, B.L., Mai, Y.-W., Ripley, M.I., Ritchie, R.O. (Eds.), Advances in Fracture Research. Pergamon Press, New York, p. 1.

Koiter, W.T., 1964. Couple-stresses in the theory of elasticity. I and II, Proc. Roy. Netherlands Acad. Sci. B67, 17.

Lloyd, D.J., 1994. Particle reinforced aluminum and magnesium matrix composites. Int. Mater. Rev. $39,1$.

Ma, Q., Clarke, D.R., 1995. Size dependent hardness in silver single crystals. J. Mater. Res. 10, 853.

McElhaney, K.W., Vlassak, J.J., Nix, W.D., 1998. Determination of indenter tip geometry and indentation contact area for depth-sensing indentation experiments. J. Mater. Res. 13, 1300.

Mindlin, R.D., 1963. Influence of couple-stress on stress concentrations. Exp. Mech. 3, 1.

Mindlin, R.D., 1964. Microstructure in linear elasticity. Arch. Rational Mech. Abal. 16, 51.

Muhlhaus, H.B., Aifantis, E.C., 1991. The influence of microstructure-induced gradients on the localization of deformation in viscoplastic materials. Acta Mechanica 89, 217.

Naghdi, P.M., Srinivasa, A.R., 1993a. A dynamical theory of structured solids. I basic developments. Phil. Trans. R. Soc. Lond. A345, 425.

Naghdi, P.M., Srinivasa, A.R., 1993b. A dynamical theory of structured solids. II special constitutive equations and special cases of the theory. Phil. Trans. R. Soc. Lond. A345, 459.

Naghdi, P.M., Srinivasa, A.R., 1994. Some general results in the theory of crystallographic slip. ZAMP $45,687$.

Nilsson, C., 1998. On nonlocal rate-dependent plasticity. Int. J. Plast 14, 551.

Nix, W.D., 1989. Mechanical properties of tin films. Metall. Trans. 20A, 2217.

Poole, W.J., Ashby, M.F., Fleck, N.A., 1996. The role of strain gradients in grain size effect for polycrystals. J. Mech. Phys. Solids 44, 465.

Schaefer, H., 1967. Das Cosserat-Kontinuum. Z. Anew. Math. Mech. 47, 485.

Shizawa, K., Zbib, H.M., 1999. A thermodynamical theory of gradient elastoplasticity with dislocation density tensor I: fundamentals. Int. J. Plast. 15, 899. 
Shu, J.Y., 1998. Scale-dependent deformation of porous single crystals. Int. J. Plast. 14, 1085.

Shu, J.Y., Fleck, N.A., 1999. Strain gradient crystal plasticity: size-dependent deformation of bicrystals. J. Mech. Phys. Solids 7, 297.

Shu, J.Y., Barlow, C.Y., 2000. Strain gradient effects on microscopic strain field in a metal matrix composite. Int. J. Plast. 16, 563.

Smyshlyaev, V.P., Fleck, N.A., 1996. The role of strain gradients in the grain size effect for polycrystals. J. Mech. Phys. Solids 44, 465.

Stolken, J.S., Evans, A.G., 1998. A microbend test method for measuring the plasticity length scale. Acta Mater. 46, 5109.

Toupin, R., 1962. Elastic materials with couple-stresses. Arch. Rational Mech. Anal. 11, 385. 